\title{
Cognitive implications of facilitating echoic persistence
}

\author{
Carryl L. Baldwin \\ Old Dominion University, Norfolk, Virginia
}

\begin{abstract}
Seventeen participants performed a tone-pattern-matching task at different presentation levels while concurrently engaged in a simulated-driving task. Presentation levels of 60, 65, and $70 \mathrm{dBC}$ (SPL) were combined factorially with tone-matching delays of 2, 3, and $4 \mathrm{sec}$. Intensity had no effect on performance in single-task conditions and short-delay conditions. However, when the participants were engaged concurrently in the driving task, a significant interaction between presentation level and delay was observed. In the longest delay condition, the participants performed the tone-pattern-matching task more efficiently (more quickly and without additional errors) as presentation intensity increased. These findings demonstrate the interaction between sensory and cognitive processes and point to a direct-intensity relationship where intensity affects the persistence of echoic memory. Implications for facilitating auditory processing and improving auditory interfaces in complex systems (i.e., transportation environments), particularly for older and hearing-impaired listeners, are discussed.
\end{abstract}

Humans are frequently required to process speech and other complex auditory information while concurrently performing other tasks that can load working memory capacity and other processing resources. Although we routinely process speech with little apparent effort in frequent daily conversations, many factors can constrain the effectiveness of speech perception, including noise, other auditory distractors, and the requirement to perform other tasks simultaneously, both visual and auditory. New technologies (i.e., mobile phones and in-vehicle auditory route guidance systems) are also making speech and other auditory stimuli increasingly prevalent in work environments, at home, and in transportation. Because auditory stimuli must frequently be processed while humans are concurrently engaged in other tasks, such as driving, it is of interest to understand how auditory presentation intensity affects echoic persistence under dualtask conditions.

Intensity and other physical features of sounds can significantly affect how efficiently auditory stimuli are processed under dual-task conditions. For example, Baldwin and Struckman-Johnson (2002) demonstrated that the presentation amplitude of auditory information affects auditory processing even when amplitudes are within a clearly audible range-45-70 dB (SPL). When concurrently performing a sentence-processing and a simulateddriving task, the participants made more errors and took longer to respond as the presentation amplitude of the auditory task decreased from 55 to $45 \mathrm{~dB}$ (SPL). A similar decrement in auditory performance was observed when an auditory mental arithmetic task was combined with a simulated-driving task (Baldwin, 2001). The ability of the participants to perform mental calculations to numbers presented auditorily declined significantly in dual-task conditions as stimulus presentation intensity decreased from 65 to $60 \mathrm{~dB}$. The participants in both of Baldwin's investigations possessed normal hearing abilities. Persons with elevated hearing thresholds (e.g., older populations) may have even greater difficulty processing complex auditory information, particularly in dual-task situations. Hearing difficulties may become particularly evident in dual-task situations and may exacerbate or be mistaken for cognitive impairments (Baldwin, 2002; Schneider \& Pichora-Fuller, 2000). Despite convincing evidence that increasing stimulus intensity in dual-task situations facilitates performance (Baldwin, 2001; Baldwin \& StruckmanJohnson, 2002), the mechanism behind this performance enhancement is less clear.

Auditory processing requires sensory stimuli to be held temporarily until meaning can be assigned to the incoming information. Several information-processing models postulate that acoustic stimuli first enter a transient sensory buffer before being processed in short-term or working memory (Atkinson \& Shiffrin, 1968; Baddeley, 1992; Baddeley \& Hitch, 1974; Norman \& Bobrow, 1975; Waugh \& Norman, 1965). Further processing leads to pattern recognition or semantic analysis of the auditory stimulus on the basis of information held in more permanent long-term memory (Altmann, 1990; Corso, 1981; Neisser \& Beller, 1965).

Considerable research has examined the nature and duration of information in the initial auditory store,

C. L. Baldwin, cbaldwin@gmu.edu 
or echoic memory (Cowan, 1984; Darwin, Turvey, \& Crowder, 1972; Moray, Bates, \& Barnett, 1965; Neisser, 1967). In an important review, Cowan pointed out that the existing literature leads to the theoretical conclusion that there are two forms of auditory sensory memory. According to Cowan's model, a short auditory store of up to $300 \mathrm{msec}$ retains stimulus properties for immediate unanalyzed recognition purposes. A longer auditory store lasting several seconds allows integration of the acoustic pattern with contextual information for recognition of stimuli that cannot be interpreted from the immediate information (i.e., ambiguous words in a sentence or comparison between sequential acoustic patterns). The duration of this longer auditory sensory storagehereafter, called echoic memory — has been found to last at least 2-4 sec (Cowan, 1984; Crowder, 1982; Darwin et al., 1972).

Despite the large body of work on echoic memory, to date, few if any investigations have explored the impact of auditory intensity on the strength or duration of echoic persistence. Such an influence seems likely, given that the effects of intensity on sensory memory have been demonstrated in vision. The effects of intensity on visual persistence are complex, and a multitude of paradigms have been used, often with equivocal results. When stimulus luminance is sufficient to produce a retinal afterimage, greater intensity increases visual persistence, a phenomenon known as the direct-intensity effect. However, at luminance levels within the mesopic visual range, the intensity of visual stimuli appears to have an inverse effect on visual persistence. That is, within the range of mesopic vision, increases in stimulus intensity are associated with decreased visual persistence. For a review of the effects of intensity on visual persistence, see a discussion in Di Lollo and Bischof (1995). Auditory stimulus intensity may influence echoic persistence in similar ways.

Echoic memory is critical to understanding speech and other forms of complex auditory processing (Fischler, 1998; Massaro, 1981; Stine, Soederberg, \& Morrow, 1996). Corso (1981) noted that initial stages of speech perception rely on the ability to discriminate between small changes in frequency or pitch, a skill that older hearingimpaired listeners often have difficulty with. Later stages rely on the ability to integrate successively heard words, phrases, and sentences with previously stored information (Pichora-Fuller, Schneider, \& Daneman, 1995). Because of the transient nature of auditory stimuli, a brief temporary storage capability is essential to maintaining information from early to later stages. Particularly when the task of auditory processing must be time-shared with a concurrent task, an echoic memory of longer duration will increase the probability that the auditory stimulus will still be available for analysis when attentional resources become available for processing.

The present investigation was designed to examine the relationship between auditory stimulus intensity and echoic persistence during concurrent performance of a simulated-driving task. It was hypothesized that present- ing auditory stimuli at a higher decibel level (in the range of 60-75 dBC SPL) would increase the duration of the echoic trace, resulting in faster and more accurate performance when participants were required to match two auditory patterns separated by a delay near the upper temporal limit of their echoic memory (4 sec, as compared with $2 \mathrm{sec}$ ).

\section{METHOD}

\section{Participants}

Seven males and 10 females (mean age, 24.59 years) with little or no formal musical training voluntarily participated in the investigation. The participants were provided with University-approved informed consent documents. The participants were screened for possible hearing deficits with a self-report questionnaire and an informal auditory-screening test using sample stimuli. One female participant was excluded from subsequent data analysis due to a suspected hearing deficit detected in the screening process.

\section{Procedure}

The participants filled out a questionnaire assessing hearing abilities, occupational and recreational noise exposure, and prior experience with driving simulation video games. On the basis of their self-reported level of experience with driving simulation games, the participants were grouped into novice and experienced groups and were assigned accordingly to either an easy or a moderately challenging simulated-driving task. The participants were then provided single-task practice with the simulated-driving task and then the tone-matching task, followed by dual-task practice with both tasks.

Auditory task. The tone-pattern-matching task required the participants to judge the similarity of a test tonal pattern to a comparison pattern and to verbally respond "same" or "different." Tone patterns consisted of five randomly selected notes from the middle $\mathrm{C}$ octave on an electronic keyboard set to synthesize a piano (see Figure 1 for a schematic illustration of the auditory task paradigm). The same tone frequency was never repeated more than once per five-note pattern. Random selection of notes resulted in tone patterns that tended to be nonharmonic. That is, the tone patterns did not represent familiar melodies, did not consist of major or minor chord structures, and did not conform to the syntactic rules of Western European music (Belin et al., 1998). Tone patterns were chosen, rather than speech stimuli, in an effort to dissociate the temporal aspects of echoic memory from phonological or linguistic cognitive operations (Belin et al., 1998).

Presentation levels (PLs) of 60, 65, and $70 \mathrm{~dB}$ SPL were used to present the tones in each condition. A delay of 2, 3, or $4 \mathrm{sec}$ separated the test and the comparison patterns. The participants were given $2 \mathrm{sec}$ to make a verbal response of "same" or "different." All the responses were recorded via a microphone for offline analysis of errors and reaction times (RTs). Tones within each pattern were presented for $50 \mathrm{msec}$ at a rate of one tone every $500 \mathrm{msec}$. RT was measured as the time between presentation of the last tone in the comparison pattern and the participants' verbal response. If no response was made prior to the onset of the next set, the set was scored as an error of omission. Errors of commission were defined as incorrect responses within the 2-sec response period. The participants performed the tone-pattern-matching task at each of the three delays at each of the three PLs in randomized order. In addition, the participants performed three baseline conditions, one at each of the three PLs at the 3-sec delay. Presentation order of the baseline conditions was counterbalanced across participants, and a baseline condition was performed at the beginning of the dual-task experimental blocks and, again, after completion of three and six experimental blocks. 


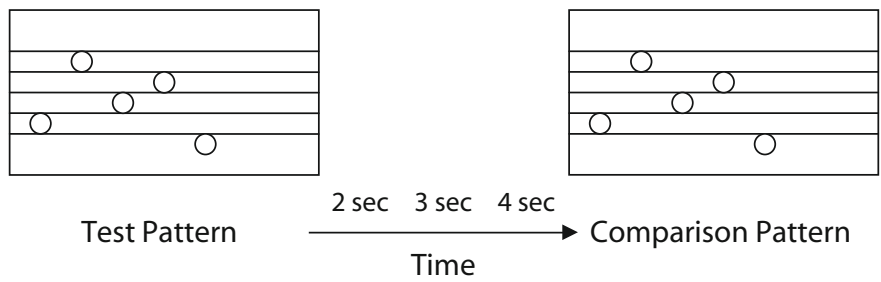

Figure 1. Schematic illustration of the auditory tone pattern-matching task. Delays of 2, 3, or 4 sec separated the test and comparison five-note tone patterns.

Driving task. A low-fidelity simulated-driving task was used so that the effects of tone intensity on echoic persistence could be examined under conditions in which the participant's processing resources were relatively loaded. The participants used steering, accelerator, and brake controls to maneuver through a simulated course at one of two difficulty levels, as deemed appropriate by their self-reported level of skill and experience with similar video game driving tasks. The experimenter verified that the participants were assigned to the appropriate difficulty level by observing their ability to reach criterion levels of performance in baseline conditions. Adjustments were made (participants were assigned to the lower difficulty level) if they could not reach criterion levels of simulated-driving performance after two practice trials. Criterion performance was defined as completing a single-task driving run with no crashes and fewer than two minor or major lane deviations while maintaining speed within the range of 20-35 mph. The participants were then allowed to practice until their simulateddriving performance reached asymptotic levels. Those participants unfamiliar with simulated-driving tasks such as the one used here exhibited improved performance over the course of the first several practice trials. Asymptotic level was defined as the point at which criterion performance was achieved, with no further observations of improvement in performance.

Tasks were operationally designated as the primary and the loading tasks through repeated instructions to the participants. That is, the participants were given instructions at several points during practice and prior to beginning the experimental trials that the auditory task was the primary, or most important, task. They were instructed that they should keep continually engaged in the driving task in order to maintain their driving performance within the specified range but to devote the majority of their attention to performing the auditory task. The participants were provided with single-task practice on the loading/driving task, followed by single-task practice on the primary tone-pattern-matching task and then the dual-task practice prior to performing in the experimental trials.

\section{RESULTS}

Performance in the simulated-driving/loading task and for the tone-pattern-matching task was analyzed in separate 3 (delay of 2,3 , or $4 \mathrm{sec}) \times 3(\mathrm{PL}$ of 60,65 , or $70 \mathrm{~dB}$ ) repeated measures MANOVAs. Both delay and PL were within-subjects factors. The Wilks's lambda statistic was used for all multivariate statistical analyses. Mauchly's $W$ was used to test for sphericity, and the GreenhouseGeisser statistic was used for univariate analyses if sphericity assumptions were violated. An alpha level of .05 was used for determining statistical significance for all the analyses, with Bonferroni alpha-level corrections made for pairwise comparisons of levels of individual dependent measures. Confidence intervals (CIs) were calculated using Loftus and Masson's (1994) formula for within-subjects designs.

\section{Driving Task}

Dependent measures of the simulated-driving/loading task included driving errors such as minor lane deviations, major deviations, driving off the road, loss of vehicular control, and crashes. Minor lane deviations were defined as occurrences in which either tire crossed the lane line demarcation on the same side (i.e., the left front tire crossed the left lane line demarcation or the right front tire crossed the right line lane demarcation). Major lane deviations were defined as occurrences in which the simulated vehicles' centerline crossed the lane line demarcation on either side (e.g., the simulated vehicle was driven onto the shoulder, so that the center of the simulated vehicle crossed the right lane line demarcation or the centerline of the vehicle crossed into the left lane line demarcation into the opposing traffic lane). Loss of vehicular control was defined as occurrences in which the driver experienced more than one major lane deviation within any one instance (the center of the simulated vehicle either crossed over the left and then the right lane line demarcation or vice versa without crashing). The multivariate analysis using a Wilks's lambda statistic revealed a significant main effect for the tone-matching delay condition $\left[F(8,54)=2.43, p<.05\right.$, partial $\eta^{2}=$ .26]. Univariate analyses revealed that minor lane deviations contributed primarily to the significant multivariate main effect for delay $[F(2,30)=5.02, p<.05$, partial $\left.\eta^{2}=.25\right]$. Assumptions of sphericity were not violated for this effect [Mauchly's $W(2)=.956, p=.732$ ]. Drivers made significantly more minor lane deviations when comparing tonal patterns in the 2 -sec delay condition $(M=2.35, S E=0.60)$, relative to the 4-sec delay condition $(M=1.65, S E=0.42)$. The 3 -sec delay condition $(M=1.62, S E=0.52)$ was not reliably different from either the 2- or the 4-sec delay condition.

Driving performance was not affected by PL, nor did any of the dependent measures of driving performance reveal a significant interaction between PL and delay.

\section{Tone-Matching Task}

Baseline trials. Auditory-task-only baseline trials were analyzed in a repeated measures MANOVA with PL as 
the factor and with RT, errors of omission, and errors of commission as dependent variables. The multivariate test revealed no significant differences between the three PL conditions $\left[F(6,56)=0.426, p>.05\right.$, partial $\left.\eta^{2}=.04\right]$. Univariate analyses of each of the dependent variables also indicated no significant differences between tonematching performance in the baseline conditions as a function of PL.

Dual-task trials. Performance in the dual-task trials (auditory-tone-matching task and concurrent simulated driving) was analyzed using a $3(\mathrm{PL}) \times 3$ (delay) repeated measures MANOVA with PL and delay as within-subjects variables. The auditory-task-dependent measures were RT, errors of omission (when no response was made before the end of the trial), and errors of commission (when an incorrect response was made).

Presentation level $\times$ delay interaction. As was predicted, a significant multivariate interaction between PL and delay condition was observed $[F(12,153)=1.82, p<$ .05 , partial $\eta^{2}=.11$, observed power $\left.=.814\right]$. Univariate analyses revealed that the dependent measure of RT to the tone-pattern-matching task was the only dependent measure that contributed to the significant multivariate interaction. For the delay $\times$ PL interaction, sphericity could be assumed for the dependent measure of RT [Mauchly's $W(9)=.906$ ] Univariate analyses revealed a significant delay $\times$ PL interaction for RT $\left[F(4,60)=3.69, p<.05\right.$, partial $\eta^{2}=.20$, observed power $=.856]$. Figure 2 illustrates the mean and CI $(95 \%)$ in each delay $\times$ PL condition. Of particular theoretical interest for the present study, in the longest delay condition ( $4 \mathrm{sec}$ ), RT increased as PL decreased. In the 4-sec delay condition, RT was significantly shorter when the stimuli were presented at a 70-dB PL, relative to when the stimuli were presented at 65- and 60-dB PLs.

There were no reliable differences in comparisons of $\mathrm{RT}$ as a function of PL in the 3-sec delay condition. In the 2-sec delay condition, RT in the 60-dB PL condition was significantly longer than $\mathrm{RT}$ in the $65-\mathrm{dB}$ condition. There were no other significant contrasts in the 2-sec delay condition. No other dependent measures were found to contribute significantly to the delay $\times$ PL interaction.
A multivariate main effect for PL approached significance $\left[F(6,56)=2.24, p=.052\right.$, partial $\left.\eta^{2}=.19\right]$. Univariate analyses revealed that the RT dependent measure for the tone-pattern-matching task primarily contributed to the marginally significant multivariate main effect. However, the univariate analysis of RT, using the Greenhouse-Geisser statistic, failed to reach significance for the main effect of PL condition $[F(2,30)=2.59, p>$ .05 , partial $\left.\eta^{2}=.14\right]$. The main effect for delay was not significant.

\section{DISCUSSION}

Echoic memory is critical to auditory processing, and the duration of this temporary sensory store may have particular relevance during the performance of concurrent tasks. In many everyday tasks, such as driving while processing auditory information, the duration of echoic storage may have important safety consequences. Therefore, understanding the potential impact of auditory presentation intensity on echoic persistence under dual-task conditions is of particular interest. The present results provide initial support for a direct-intensity effect on echoic memory, so that increases in presentation intensity result in echoic memory traces of longer duration. As has been demonstrated in the present investigation, in the longest delay conditions (4 sec, as compared with 3 and $2 \mathrm{sec}$ ), the participants were able to make correct comparisons between tone patterns significantly more quickly when the stimuli were presented at higher intensities $(70 \mathrm{~dB}$, relative to 65 and $60 \mathrm{~dB}$ ), without notable disruptions to simulated-driving performance.

An important feature of the present results is that the influence of auditory intensity on memory function was not due to near-threshold effects. PL did not affect tonematching performance in the baseline conditions, thus confirming that all the auditory stimuli were presented within a clearly audible range in all the conditions. However, when the participants were required to perform both the simulated-driving task and the auditory task, higher PLs facilitated performance. Previous research has indi-

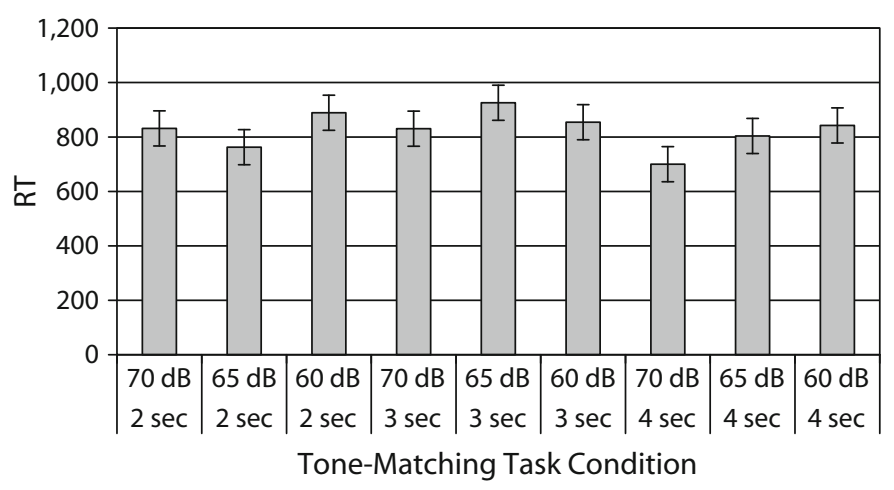

Figure 2. Mean response times (RTs, in milliseconds) as a function of tone-pattern-matching task condition. Error bars represent $95 \%$ confidence intervals calculated for a within-subjects design. 
cated that the temporal limit of echoic memory is roughly $4 \mathrm{sec}$ (Darwin et al., 1972; Watkins \& Watkins, 1980) and that, within this time frame, the echoic memory trace begins to fade. Similar to the direct-intensity effect observed in the visual modality (Di Lollo \& Bischof, 1995), auditory PLs of increased intensity may have a direct effect on the duration of the echoic memory trace. The effect of intensity was observed in the longest delay condition (4 sec) and did not affect performance in single-task baseline conditions or in the shorter 3-sec delay condition. This finding provides initial support for the position that intensity affects echoic persistence, rather than merely ensuring that stimuli are more discernible. If the benefits of higher intensity levels were a result of stimuli's simply being more discernible, performance benefits would be expected in baseline, as well as short-delay, conditions (Di Lollo \& Bischof, 1995). The present results do not support that position. However, this should not be interpreted as suggesting that stimulus intensity affects only the duration and not the quality of the echoic trace. Sensory traces are thought to degrade gradually, rather than abruptly (Atienza, Cantero, \& Gómez, 2000; Cowan, 1984). Therefore, the quality of the echoic trace would be expected to be of less discernible quality the longer the time interval between stimulus presentation and recall. Echoic trace quality would be expected to interact with trace duration. For example, a relatively weak echoic trace with a time course of only $2 \mathrm{sec}$ would be expected to be of poorer quality $1.5 \mathrm{sec}$ after presentation, relative to an echoic trace with a time course of $4 \mathrm{sec}$ at 1.5 -sec postpresentation. The observed interaction between PL and decay in the present investigation provides preliminary support for this interpretation. Examining performance as a function of stimulus intensities at even longer delays ( $5 \mathrm{sec}$ or longer) could further illuminate the relationship between trace quality and duration.

The results indicate that in terms of driving performance, the shorter delay condition was more difficult than the longer delay conditions. This is evidenced by the unexpected increase in minor lane deviations in the loading task in the 2-sec delay condition, relative to the 4-sec delay condition. This finding suggests that although the effects of PL are evidenced in dual-task conditions, the direct-intensity effect does not appear to be dependent solely on overall task-processing requirements. Rather, the effects of PL are evidenced when the auditory task requires maintenance of stimulus information over extended durations. These results support the general view that sensory factors can interact with perceptual and cognitive processing even for stimuli that are well above threshold (Baldwin, 2002).

The initial stages of speech processing rely on brief temporary storage to maintain information until decisional processes, including lexical selection, can be made at later stages (Davis, Marslen-Wilson, \& Gaskell, 2002; Marslen-Wilson, 1987; O’Rourke \& Holcomb, 2002). An echoic memory trace of longer duration may be of particular benefit when auditory stimuli are relatively ambiguous (the number of alternatives are high) or unfa- miliar or when processing resources must be shared with additional tasks. Baddeley's (1992) model of working memory includes a component referred to as the phonological store, capable of holding auditory information for 1-2 sec. The phonological store, a subcomponent of the phonological loop, is thought to be essential to speech comprehension when processing demands are high and also to facilitate learning of novel or unfamiliar sound patterns (Baddeley, 1992; Baddeley, Gathercole, \& Papagno, 1998). An echoic trace of longer duration may increase the functional capacity of the phonological store, thus facilitating speech comprehension when processing demands are high. Furthermore, the echoic trace is thought to decay, rather than extinguish abruptly. Thus, the phonological material available in the initial period of a more persistent echoic memory would be expected to be of greater clarity, relative to shorter duration traces. The greater clarity of the phonological material may decrease the mental resource requirements of lexical extraction. Models of spoken word recognition propose that multiple lexical candidates are activated by the initial word segments until alternatives can be ruled out through subsequent acoustical input (Davis et al., 2002). Accordingly, initial segments of greater ambiguity would result in activation of a greater number of lexical alternatives and, therefore, a more cognitively demanding lexical selection process. Presentation level, a sensory factor, may therefore interact with subsequent perceptual and cognitive stages of auditory processing, potentially compromising performance when task demands are high.

The interaction between sensory and perceptualcognitive processes has particular relevance for the examination of speech recognition in older, hearing-impaired listeners. Older listeners with elevated hearing thresholds are known to rely on context for speech processing more than do their younger counterparts (Marshall, Duke, \& Walley, 1996). Marshall and colleagues observed that in low-context conditions, older listeners required more of a target word's acoustic-phonetic information in order to identify the word, relative to younger listeners. Older participants took longer than did their younger counterparts to identify the initial phonemes at the beginning of stimulus words. Once phonemes were recognized and phoneme cohorts were identified, older individuals tended to compensate or make up for lost time by performing the later stages of word recognition (isolating the unique word from among lexical alternatives and accepting this identification) more quickly than did their younger counterparts. The additional cognitive effort that older listeners must apply in later stages of word recognition to compensate for initial phoneme identification difficulties could compromise performance when overall task demands are high. During periods of high task demand, a more persistent echoic trace would allow listeners greater flexibility in the allocation of limited attentional resources.

Greater flexibility in the allocation of attentional resources, due to a more persistent echoic trace, may explain the performance benefits of increased presentation intensity observed in previous investigations (Baldwin, 
2001; Baldwin \& Galinsky, 1999; Baldwin \& StruckmanJohnson, 2002). Specifically, in challenging dual-task conditions, increased speech PLs resulted in improved speech-processing performance. Presentation intensity did not affect speech processing in single-task conditions or in low-demand dual-task conditions (Baldwin \& Struckman-Johnson, 2002). The performance benefits of a more persistent echoic trace may be most apparent in hearing-impaired populations. Persons with elevated hearing thresholds may be expected to have greater difficulty processing complex auditory information, particularly in dual-task situations. A more persistent echoic trace could result in greater flexibility in the allocating of attentional resources between stages of spoken word recognition and concurrent task demands. This may partially explain why older adults with elevated hearing thresholds benefit from stimulus intensity levels well above threshold, particularly in dual-task situations.

Auditory displays are becoming increasingly prevalent in the modern automobile (Baldwin, 2002). In-vehicle routing and navigational systems (IRANS) are an example of emerging technologies that rely on auditory displays. In order to be implemented safely, drivers must be able to maintain control of the vehicle and allocate attention to the navigational guidance only when it is safe to do so. Auditory guidance systems that support flexible attention allocation can reduce crash potential.

Older drivers often have difficulty navigating through unfamiliar or complex intersections. IRANS designed in accordance with the sensory- and cognitive-processing capabilities of older drivers therefore have the potential of increasing the safety and mobility of a growing proportion of the U.S. driving population (Baldwin, 2002).

The present results provide support for a direct-intensity effect on echoic persistence. Increased auditory presentation intensity leads to a longer duration echoic trace, which, in turn, may facilitate flexible attention allocation in dual-task situations. These results support the importance of considering the interacting influences of sensory and cognitive processes when examining human performance (Baldwin, 2002). For example, during speech processing, initial word segments must be stored until potential lexical candidates can be eliminated by subsequent input and contextual cues. Initial word segments of lower intensity would result in the activation of a greater number of possible lexical candidates and a shorter temporal duration in which to make a selection. The additional attentional effort required to process low-intensity auditory information could compromise performance of additional tasks. For the driver of a motor vehicle, any attention devoted to a speechprocessing task must be taken away from the primary task of driving. Increasing the presentation intensity of auditory route guidance and other in-vehicle displays may decrease their attentional costs and facilitate driver safety.

\section{AUTHOR NOTE}

This work was supported by the National Institutes of Health, National Institute of Aging (NIH-NIA), Grant 5 R03 Ag 23881-01. Much gratitude goes to Eric $\mathrm{H}$. Williams for his assistance in stimulus construction and data collection. Correspondence concerning this article should be addressed to C. L. Baldwin, Department of Psychology, George Mason University, Fairfax, VA 22030 (e-mail: cbaldwin@gmu.edu).

$$
\begin{array}{r}
\text { Note-This article was accepted by the previous editorial team, } \\
\text { when Colin M. MacLeod was Editor. }
\end{array}
$$

\section{REFERENCES}

Altmann, G. T. M. (1990). Cognitive models of speech processing: An introduction. In G. T. M. Altmann (Ed.), Cognitive models of speech processing: Psycholinguistic and computational perspectives (pp. 483-512). Cambridge, MA: MIT Press.

Atienza, M., Cantero, J. L., \& Gómez, C. M. (2000). Decay time of the auditory sensory memory trace during wakefulness and REM sleep. Psychophysiology, 37, 485-493.

AtKinson, R. C., \& Shiffrin, R. M. (1968). Human memory: A proposed system and its control processes. In K. W. Spence \& J. T. Spence (Eds.), The psychology of learning and motivation: Advances in research and theory (Vol. 2, pp. 89-195). New York: Academic Press.

BADDELEY, A. D. (1992). Working memory. Science, 255, 556-559.

Baddeley, A. [D.], Gathercole, S., \& Papagno, C. (1998). The phonological loop as a language learning device. Psychological Review, 105, 158-173.

Baddeley, A. D., \& Нitch, G. (1974). Working memory. In G. H. Bower (Ed.), The psychology of learning and motivation: Advances in research and theory (Vol. 8, pp. 47-89). Orlando, FL: Academic Press.

BALDWIN, C. L. (2001, October). Impact of age-related hearing impairment on cognitive task performance. Paper presented at the 45th Annual Conference of the Human Factors and Ergonomics Society, Minneapolis, MN.

BaLdwin, C. L. (2002). Designing in-vehicle technologies for older drivers: Application of sensory-cognitive interaction theory. Theoretical Issues in Ergonomics Science, 3, 307-329.

Baldwin, C. L., \& Galinsky, A. M. (1999). Pure-tone threshold shifts during moderate workload conditions: Human performance implications for automated environments. In M. W. Scerbo \& M. Mouloua (Eds.), Automation technology and human performance: Current research and trends (pp. 296-300). Mahwah, NJ: Erlbaum.

Baldwin, C. L., \& Struckman-Johnson, D. (2002). Impact of speech presentation level on cognitive task performance: Implications for auditory display design. Ergonomics, 45, 61-74.

Belin, P., Zilbovicius, M., Crozier, S., Thivard, L., Fontaine, A., Masure, M.-C., \& SAMSON, Y. (1998). Lateralization of speech and auditory temporal processing. Journal of Cognitive Neuroscience, $\mathbf{1 0}$, 536-540.

Conso, J. F. (1981). Aging sensory systems and perception. New York: Praeger.

Cowan, N. (1984). On short and long auditory stores. Psychological Bulletin, 96, 341-370.

Crowder, R. G. (1982). Decay of auditory memory in vowel discrimination. Journal of Experimental Psychology: Learning, Memory, \& Cognition, 8, 153-162.

Darwin, C. J., Turvey, M. T., \& Crowder, R. C. (1972). An auditory analogue of the Sperling partial report procedure: Evidence for brief auditory storage. Cognitive Psychology, 3, 255-267.

Davis, M. H., Marslen-Wilson, W. D., \& Gaskell, M. G. (2002). Leading up the lexical garden path: Segmentation and ambiguity in spoken word recognition. Journal of Experimental Psychology: Human Perception \& Performance, 28, 218-244.

Di Lollo, V., \& Bischof, W. F. (1995). Inverse-intensity effect in duration of visible persistence. Psychological Bulletin, 118, 223-237.

Fischler, I. (1998). Attention and language. In R. Parasuraman (Ed.), The attentive brain (pp. 381-399). Cambridge, MA: MIT Press.

Loftus, G. R., \& Masson, M. E. J. (1994). Using confidence intervals in within-subject designs. Psychonomic Bulletin \& Review, 1, 476-490.

Marshall, N. B., Duke, L. W., \& Walley, A. C. (1996). Effects of age and Alzheimer's disease on recognition of gated spoken words. Journal of Speech \& Hearing Research, 39, 724-733. 
Marslen-Wilson, W. D. (1987). Functional parallelism in spoken word-recognition. Cognition, 25, 71-102.

Massaro, D. W. (1981). Sound to representation: An informationprocessing analysis. In T. Myers, J. Laver, \& J. Anderson (Eds.) The cognitive representation of speech (pp. 181-192). Amsterdam: North-Holland.

Moray, N., Bates, A., \& Barnett, T. (1965). Experiments on the four-eared man. Journal of the Acoustical Society of America, 38, 196-201.

NeIsser, U. (1967). Cognitive psychology. Englewood Cliffs, NJ: Prentice Hall.

Neisser, U., \& Beller, H. K. (1965). Searching through word lists. British Journal of Psychology, 56, 349-358

Norman, D. A., \& Bobrow, D. G. (1975). On data-limited and resourcelimited processes. Cognitive Psychology, 7, 44-64.

O'Rourke, T. B., \& Holcomb, P. J. (2002). Electrophysiological evidence for the efficiency of spoken word processing. Biological Psychology, 60, 121-150.

Pichora-Fuller, M. K., Schneider, B. A., \& Daneman, M. (1995).
How young and old adults listen to and remember speech in noise. Journal of the Acoustical Society of America, 97, 593-608.

Schneider, B. A., \& Pichora-Fuller, M. K. (2000). Implications of perceptual deterioration for cognitive aging research. In F. I. M. Craik \& T. A. Salthouse (Eds.), The handbook of aging and cognition (2nd ed., pp. 155-219). Mahwah, NJ: Erlbaum.

Stine, E. A. L., Soederberg, L. M., \& Morrow, D. G. (1996). Language and discourse processing through adulthood. In F. BlanchardFields \& T. M. Hess (Eds.), Perspectives on cognitive change in adulthood and aging (pp. 255-290). New York: McGraw-Hill.

Watkins, O. C., \& Watkins, M. J. (1980). The modality effect and echoic persistence. Journal of Experimental Psychology: General, 109, 251-278.

Waugh, N. C., \& Norman, D. A. (1965). Primary memory. Psychological Review, 72, 89-104.

(Manuscript received May 24, 2004; revision accepted for publication July 15,2005 .) 